\title{
UNA PROPUESTA METODOLOGICA PARA EL ESTUDIO DEL PAISAJE INTEGRADO
}

\author{
Paloma IBARRA BENLLOCH \\ Dpto. de Geografía y Ordenación del Territorio \\ Universidad de Zaragoza
}

\begin{abstract}
Resumen: Se presenta una metodología para el estudio del paisaje integrado apoyada en una concepción del paisaje geográfica y sistémica. El estudio se plantea a escalas medias $(1 / 25.000-1 / 50.000)$ y consta de cuatro grandes etapas: $1^{a}$ ) Análisis de los elementos del paisaje; $2^{a}$ ) Delimitación y caracterización de las unidades de paisaje; $3^{a}$ ) Factores explicativos del paisaje; $4^{a}$ ) Diagnóstico sobre la dinámica del paisaje.
\end{abstract}

Palabras clave: Paisaje integrado, unidades de paisaje, descripción, explicación y dinámica del paisaje.

Abstract: A methodology to the study of the integrated landscape is shown, based on a geographical and systematic conception of the landscape. The study is thought at half scale $(1 / 25.000-1 / 50.000)$ and is composed of four big stages: $\left.1^{\mathrm{a}}\right)$ The landscape elements analysis; $2^{\mathrm{a}}$ ) Delimitation and characterisation of the landscape units; $3^{\mathrm{a}}$ ) Explanatory landscape factors; $4^{\mathrm{a}}$ ) Diagnosis about the landscape dynamic.

Key words: Integrated landscape, landscape units, characterisation, explanation and dynamic of the landscape.

\section{PREAMBULO}

Partimos del interés creciente que pueden y deben tener los estudios de un territorio desde un punto de vista paisajístico integrado, tanto por lo que aportan en sí mismos, como por el marco de referencia que posibilitan de cara a posteriores estudios más especializados, sin olvidar su aplicación en cuestiones de Ordenación del Territorio y Estudios de Impacto Ambiental. Somos conscientes de la problemática derivada de las múltiples acepciones y usos del término "paisaje" y de los variados 
enfoques, métodos y contenidos que pueden llegar a tener estudios igualmente denominados de paisaje. Todo ello crea un cierto confusionismo y ha contribuido a devaluar el término paisaje y los estudios paisajísticos. En buena parte, es responsabilidad de la comunidad de geógrafos clarificar los conceptos, utilizar bien la terminología y, sobre todo, realizar estudios de paisaje sólidamente construidos, que contribuyan a cubrir el "vacío" existente en el conocimiento de nuestro territorio desde esta óptica.

En este contexto, nuestra aportación consiste en presentar una metodología para el estudio del paisaje integrado que se apoya en una concepción del paisaje geográfica y sistémica. El método propuesto responde al objetivo general de realizar un estudio de paisaje integrado a una escala media $(1 / 25.000,1 / 50.000)$ y desde la Geografía, aunque podría también ser aplicado por un equipo interdisciplinar. Ha sido ensayado en el Sur del Campo de Gibraltar (Ibarra, 1993) pero pensamos que es un método suficientemente flexible como para ser aplicado a espacios de características muy diferentes. El proceso metodológico se divide en cuatro grandes etapas y es el siguiente:

\section{INTRODUCCION Y ANALISIS DE LOS ELEMENTOS DEL PAISAJE (FASE I)}

\section{1.- Planteamiento de objetivos y principios metodológicos}

En la línea de las escuelas alemana, soviética, polaca y francesa y de autores como Troll (1939, 1950), Neef (1963), Haase (1964), Sochava (1963), Isachenko (1978), Tricart y Kilian (1979), Bertrand $(1968,1969,1972 \ldots)$, entendemos el paisaje desde un punto de vista geográfico y sistémico; como una proyección en un territorio dado de lo que Sochava (1963) definió como "geosistema", ligando más al territorio el concepto de "ecosistema" de Tansley (1935). Un geosistema se define como un sistema de relaciones geográficas compuesto de un fenosistema o conjunto de formas externas que estructuran y caracterizan un territorio y de un criptosistema o sistema oculto, complemento imprescindible del fenosistema, pues proporciona la explicación precisa para la comprensión del geosistema (Gonzalez Bernaldez, 1981).

Así, el fenosistema está formado por los denominados elementos del paisaje: la litología, el relieve, el suelo, el agua y la red hidrográfica en que se localiza y la cubierta del suelo, que incluye como componentes "estáticos" la cubierta vegetal y la no vegetal, construida o creada por el hombre, y como componentes "móviles" a la fauna y al hombre (en cuanto a su presencia, no a su actividad). Un estudio de paisaje integrado no debe limitarse al análisis y descripción del fenosistema sino que debe abordar también las conexiones entre éste y el criptosistema, es decir, entre las formas 
que observamos y los factores que las explican y condicionan su dinámica. El criptosistema se concreta en los denominados factores del paisaje que son de tipo climático, geológico y antrópico y actúan movidos por las fuentes de energía que entran en el geosistema: la energía solar, la gravedad, las fuerzas internas de la tierra y la propia energía liberada por los seres vivos, principalmente por el hombre.

De acuerdo con estos planteamientos es sumamente interesante el concepto de unidad de paisaje, en la línea en que Bertrand (1968, 1969, 1972...) lo plantea, inspirado en las escuelas de paisaje de la antigua República Democrática Alemana y de la Unión Soviética y, también, en la escala espacio-temporal propuesta por Tricart y Cailleux (1956) para las unidades geomorfológicas, adoptada por él para proponer una taxonomía de unidades de paisaje. Entendemos las unidades de paisaje como espacios que, a una escala determinada, se caracterizan por una fisionomía homogénea y una evolución común, siendo de unas dimensiones concretas y cartografiables. El proceso de delimitación de estas unidades, basado en criterios seleccionados a partir de las especiales características y combinaciones de los distintos elementos del paisaje, es uno de los aspectos metodológicos que más hay que cuidar con objeto de no caer en un exceso de subjetivismo que invalide los resultados obtenidos y que, sin duda, va en contra de la construcción de la ciencia del paisaje que deseamos.

Bertrand da gran importancia al aspecto dinámico de las unidades de paisaje. Basándose en la teoría bio-rexistásica de Ehrart (1956), plantea una tipología dinámica que clasifique a las unidades de paisaje en función del sistema de evolución, del estado alcanzado por analogía con el clímax y del sentido general de la dinámica (progresiva, regresiva y estable). Coincidimos básicamente en estos planteamientos aunque no tanto con la forma fundamentalmente cualitativa con que los aplica este autor. Por otro lado, es preciso complementarlos con los planteamientos de Tricart y Kilian (1979) que defienden que la dinámica del paisaje depende fundamentalmente del balance entre morfogénesis y edafogénesis, balance que puede variar en el tiempo y en el espacio. La metodología que proponemos ha tenido especial cuidado en posibilitar alcanzar un diagnóstico sobre la dinámica de las unidades de paisaje.

En consecuencia, como objetivos más concretos a conseguir en un estudio de paisaje a escalas medias, se proponen los siguientes:

- Delimitación de unidades homogéneas a los niveles $\mathrm{V}^{1}\left(10-100 \mathrm{~km}^{2}\right)$ y VI (1 $10 \mathrm{~km}^{2}$ ); cartografiarlas y caracterizarlas integrando y relacionando sus elementos. $\mathrm{La}$ elección de estos niveles se apoya en que son escalas que recogen bien la diversidad existente en el paisaje y permiten establecer relaciones coherentes entre sus elementos,

1 Según la taxonomía de Tricart y Cailleux (1956) 
así como un diagnóstico sobre su dinámica, resultando, además, unidades cartografiables de escalas útiles de cara a la ordenación del territorio y posibles estudios de impactos.

- Explicación de los rasgos fundamentales del paisaje apoyándose en las interacciones detectadas entre los factores y elementos de mayor peso en el mismo.

- Establececimiento de un diagnóstico sobre la dinámica de las distintas unidades de paisaje.

\section{2.- Elección y delimitación del área de estudio}

En esta fase es preciso ser extremadamente prudente, en el sentido de valorar bien los medios y la información de que se dispone puesto que, en muchas ocasiones, la ausencia de una adecuada información de base obliga a elaborarla. Ello constituye un esfuerzo añadido que ha de influir en la extensión del área a estudiar.

3.- Recopilación de la información bibliográfica, cartográfica, fotográfica, estadística y documental referida tanto a los elementos como a los factores del paisaje

Dada la diversidad y volumen de la información necesaria es una etapa que se va completando paralelamente a las siguientes.

\section{4.- Análisis de los elementos del paisaje}

Para el estudio de cada elemento es necesario aplicar una metodología diferente, específica del propio elemento y bien definida dentro de la correspondiente ciencia (Geología, Gemorfología, Edafología, Biogeografía...), por lo que no es precisa ninguna referencia a ellas, pues son bien conocidas. Ahora bien, hay que tener muy en cuenta que el análisis de estos elementos es imprescindible como medio para alcanzar los objetivos planteados pero, aunque no esté exento de interés, no es un fin en sí mismo. Por ello habrá de profundizarse hasta el nivel necesario para lograr la integración paisajística a una escala media y para sopesar, posteriormente, hasta qué punto cada elemento interviene en la definición de las unidades de paisaje.

Lo deseable sería contar con trabajos sectoriales que proporcionasen una información ya elaborada y una cartografía adecuada, para limitarnos a revisar, comprobar y, en caso necesario, completar y reorganizar dicha información y poder abordar directamente el verdadero objetivo del trabajo. Sin embargo, en la mayoría de las ocasiones esto no ocurre y es preciso invertir tiempo y esfuerzo en conseguir la descripción, tipología y cartografía adecuadas de los distintos elementos del paisaje. El esquema metodológico general es el siguiente: 
- Revisión de la información existente

- Fotointerpretación y elaboración de cartografía cuando sea necesario

- Trabajo de campo y recogida de muestras (suelo, vegetación) cuando sea necesario

- Trabajo de laboratorio (suelo) cuando sea necesario

- Establecimiento de una tipología de cada elemento y redacción de una síntesis descriptiva de cada elemento apoyada en la cartografía

Al final de esta etapa, se debe contar con cartografía a escala 1/25.000 ó $1 / 50.000$ de todos los elementos del paisaje: mapa litológico, mapa de altitudes absolutas, mapa de pendientes (método de los intervalos móviles, Denness y Grainger, 1976), mapa geomorfológico (diferenciando elementos estructurales, unidades geomorfológicas a los niveles V, VI y VII y procesos morfogenéticos), mapa hidrográfico (método de inflexiones de curvas de nivel, Morisava, 1985), mapa de suelos $^{2}$ y mapa de cubierta del suelo (diferenciando espacios naturales -formaciones vegetales- $\mathrm{y}$ espacios antropizados).

\section{DELIMITACION Y CARACTERIZACION DE LAS UNIDADES DE PAISAJE (FASE II)}

\section{1.- Diagnóstico sobre el peso de cada elemento en la estructuración del paisaje a distintas escalas}

El diagnóstico se basa en el análisis de las discontinuidades en el paisaje a que cada elemento da lugar por medio de una tipología más o menos contrastada. Este diagnóstico tiene por objeto establecer unos criterios que sirvan para diferenciar y delimitar las unidades homogéneas. Se analiza hasta qué punto articulan el paisaje, a diferentes escalas, los distintos elementos que lo componen, a partir de la cartografía, tipologías y descripciones expuestas en la parte I. En consecuencia, se selecciona uno u otro elemento, o la combinación de varios, como criterios de delimitación de las unidades de paisaje (homogeneidad a escala V) y de las unidades ambientales (homogeneidad a escala VI) en que éstas se subdividen.

\footnotetext{
${ }^{2}$ La escasez de cartografía de suelos a estas escalas en España, y la complejidad de su elaboración, pueden obligar a limitarse a establecer una tipología de los principales suelos existentes basada en las variaciones litológicas y geomorfológicas fundamentales.
} 


\section{2.- Propuesta y delimitación provisional de unidades de paisaje y unidades ambientales}

El método utilizado para delimitar espacialmente, a escala $1 / 25.000$, las unidades de paisaje es la combinación de la información extraída de los mapas temáticos de aquellos elementos seleccionados como criterios de delimitación. Esta primera elaboración del mapa de unidades de paisaje permite detectar los problemas existentes en la delimitación, así como abordar la siguiente etapa.

\section{3.- Diseño del inventario de campo para la recogida sistemática de información sobre el terreno}

La información de una parte de las variables a analizar (tanto características del paisaje como factores influyentes sobre aquéllas) sólo puede obtenerse directamente sobre el terreno, en puntos concretos. Conviene diseñar un inventario de campo que permita recopilar esta información de forma sistemática. Este inventario contendrá tanto variables necesarias para la caracterización de las unidades de paisaje como para su explicación. Aunque la selección de algunas variables dependerá de las características específicas de cada espacio, otras tienen un carácter general. Por ello, creemos interesante enumerar las variables seleccionadas en el trabajo del Campo de Gibraltar ${ }^{3}$. Son las siguientes: variables de localización, variables geomorfológicas (altitud, orientación, posición topográfica, pendiente, presencia de grietas de retracción, de reptación, de derrubios de gravedad, de formas solifluidales, de surcos...), variables de cubierta del suelo (tipo de cubierta del suelo, estructura y composición de la vegetación -índice de abundancia- dominancia de Braun Blanquet, 19794, tanto por especie como por estrato-, diámetro medio del arbolado, enfermedades del arbolado), variables hidrológicas (caudal permanente o estacional), variables climáticas (zona de nieblas, deformación del arbolado por el viento ${ }^{5}$ ), variables antrópicas (accesibilidad/proximidad a núcleos de población, tipo de propiedad, extracción de corcho, extracción masiva de leña, roza reciente, carga ganadera en unidades lanares por Ha., incendios en la última década, incendios antiguos, roturación, caza mayor, repoblación con especies alóctonas), variables de visibilidad (desde dentro hacia fuera, desde fuera hacia dentro). Como hemos dicho,

\footnotetext{
${ }^{3}$ La diversidad de las variables y la escasez de medios, junto a la propia escala del trabajo, obligan a optar en muchos casos por variables categóricas, pero siempre que sea posible es preferible una cuantificación mayor, es decir, la utilización de variables numéricas, puesto que suponen una información más rigurosa y permiten un tratamiento estadístico de mayor calidad.

4 Indices de abundancia/dominancia: $5=$ superior al $75 \% ; 4=$ entre 50 y $75 \%$; $3=$ entre 25 y $50 \% ; 2$ = entre 10 y $25 \% ; 1=$ inferior al $10 \%$; + especies aisladas.

${ }^{5}$ Escala deBarsch (1963).
} 
el inventario incluye variables relacionadas con el proceso descriptivo de las unidades de paisaje, pero también con los factores explicativos, cuyo análisis es necesario para el desarollo de la fase que posteriormente describiremos como III.2. Por tanto en este punto deben sincronizarse las fases II y III de nuestro método, para utilizar ambas la información obtenida en el estudio de campo.

\section{4.- Diseño del muestreo para la recogida de la información sobre el terreno}

Para que la información procedente de los inventarios de puntos concretos sea representativa del conjunto del territorio, y de cada una de las unidades en que éste se divide, es preciso realizar un muestro de puntos. Un muestreo adecuado al estudio del paisaje es el estratificado, actuando como estratos las propias unidades de paisaje previamente delimitadas e incluyéndose un número de puntos de cada unidad proporcional a su extensión. Dentro de cada unidad los puntos se distribuyen cubriendo la diversidad existente. De esta manera, se obtiene una representación suficiente de las unidades de paisaje, pudiéndose conocer sus características y las diferencias entre ellas. En cuanto al tamaño de la muestra, debe alcanzarse un volumen que permita la representación de todas las unidades $y$, al mismo tiempo, quede dentro de las posibilidades reales del trabajo.

5.- Recogida de la información de los inventarios de campo que componen la muestra y comprobación de las unidades de paisaje y ambientales delimitadas

Esta etapa supone un minucioso trabajo de campo que, en el caso de ser realizado por un equipo, obliga a cuidar mucho la uniformidad de criterios en la recogida de la información. Toda esta información ha de ser adecuadamente organizada en una base de datos con el fin de facilitar su utilización y análisis estadístico en las siguientes fases del trabajo; lo idóneo sería utilizar un Sistema de Información Geográfica.

\section{6.- Delimitación cartográfica definitiva de las unidades de paisaje y de sus unidades ambientales}

Tras finalizar el trabajo de campo, se puede abordar la cartografía definitiva de las unidades de paisaje y ambientales.

\section{ambientales}

7.- Descripción de cada una de las unidades de paisaje y de sus unidades

En la descripción de cada una de las unidades delimitadas se utiliza tanto la información generada en la parte I del trabajo como la recogida en los inventarios de campo. Ha de hacerse hincapié en las relaciones entre las diferentes variables pues, precisamente, lo que caracteriza a cada paisaje es la forma peculiar que tienen de 
combinarse los diferentes elementos. Esta caracterización de las unidades de paisaje nos permite un tratamiento integrado de la información del territorio. Esta descripción, que constituye una parte fundamental del trabajo, puede ilustrarse con cuadros sintéticos de la información recogida en los inventarios de campo, como pueden ser: tablas de frecuencia de las formas de modelado presentes; listados de especies florísticas de los inventarios más representativos de las facies de las formaciones vegetales, representados mediante pirámides de vegetación según el método de Bertrand (1966); fotografías; bloques diagramas y transectos, etc...

\section{FACTORES EXPLICATIVOS DEL PAISAJE (FASE III)}

\section{1.- Detección de las relaciones entre factores y elementos del paisaje}

Esta etapa consiste en poner de relieve las principales relaciones existentes entre los factores y los elementos del paisaje. Estas relaciones son tan numerosas que podrían hacer el trabajo inabarcable, por lo que se propone la opción de limitarse a las relaciones con los elementos de mayor peso en la articulación del paisaje, que son aquellos que intervienen de forma decisiva en la delimitación de las unidades de paisaje y unidades ambientales.

\section{2.- Explicación de los elementos de mayor peso en la articulación del paisaje}

El esquema metodológico a seguir en esta etapa es el siguiente:

- Análisis de los factores intervinientes y elaboración de la información de cada factor y de sus variaciones en el territorio, con el fin de poder relacionarlos con los elementos del paisaje. Toda esta información ha de tener referencias espaciales, pero en algunos casos será conveniente, incluso, la elaboración de cartografía temática (por ejemplo; mapa de espacios de afinidad climática, mapa de propiedad, mapa de localización de incendios forestales...).

- Formulación de hipótesis explicativas de los elementos del paisaje. Hay que señalar que no siempre son precisas estas hipótesis pues, por ejemplo, hay algunos factores antrópicos como la actividad urbanizadora, la evolución demográfica y, en algunos aspectos, el factor de actividades económicas, que explican por sí mismos y de forma directa los tipos de cubierta no vegetal del suelo. la muestra.

- Confirmación de las hipótesis a partir de la información de los inventarios de 
En el ámbito del territorio español, tanto el relieve como la cubierta del suelo se configuran como elementos que suelen tener un papel importante en la organización de los paisajes, por lo que se expone a continuación el proceso a seguir en la explicación de dichos elementos, sin que ello signifique que sean siempre los principales o los únicos.

2.1.- La explicación del relieve actual se apoya en la de los factores que lo condicionan en mayor medida: la explicación de los materiales constituyentes, la de las fases orogénicas que han actuado sobre ellos generando su estructura y la de los procesos morfogenéticos que han modelado posteriormente dicho relieve estructural. En consecuencia, las fases a desarrollar son las siguientes:

a.- Ubicación del área de estudio dentro del contexto geológico regional.

b.- Síntesis de los procesos de formación de los materiales constituyentes y del modelo paleogeográfico en el que se sitúan estos procesos.

c. - Hipótesis explicativas de la evolución geológica y establecimiento de las principales etapas de la evolución geológica (normalmente basadas en una revisión y síntesis de los estudios previos).

d.- Descripción de la estructura tectónica en cuanto explicativa de muchas de las formas de relieve actual.

e.- Análisis de los procesos morfogenéticos que han actuado sobre el relieve estructural y son los responsables de su modelado. Para ello se combina la caracterización morfoclimática del territorio y la información sobre la presencia de las formas de modelado en las distintas unidades ambientales procedente de los inventarios de campo. Con todo ello, se llega a explicar qué procesos y en qué forma han modelado el relieve que configura el paisaje que observamos.

2.2.-La explicación de la cubierta del suelo se apoya en los siguientes planteamientos: pensamos que para explicar la vegetación actual es conveniente partir de la vegetación climácica para poder, posteriormente, establecer las distintas etapas de degradación respecto a ella. Todo ello, asumiendo la imposibilidad o dificultad que, en espacios extremadamente modificados por el hombre, puede tener el establecimiento de la vegetación climácica, y considerando la clímax en relación con las condiciones climáticas y edáficas actuales (no tiene por qué coincidir con la clímax de tiempos pasados). De esta manera se ordenan las distintas formaciones vegetales que "crean paisaje" estableciéndose relaciones dinámicas entre las mismas. En este proceso podrán análizarse, como factores explicativos del paisaje, tanto aquellos que lo son por determinar la vegetación climácica como los que influyen en la presencia de determinadas fases de degradación respecto a aquélla. Partiendo de estos 
planteamientos, que se enmarcan en la Sinfitosociología, el proceso que se propone es el siguiente:

$1^{\mathrm{a}}$ - Análisis de los factores intervinientes, es decir, climáticos y antrópicos (sin olvidar aquellos factores como suelo o hidrología que, al ser elementos del paisaje, habrán sido analizados en fases anteriores).

$2^{a}$ - Definición de las series de vegetación ${ }^{6}$ que corresponden al espacio biogeográfico estudiado, diferenciando las series climatófilas de aquellas edafófilas. Para ello, se combina la información de los espacios de afinidad climática caracterizados bioclimáticamente, del tipo de suelo, de la presencia de agua en el suelo y, naturalmente, de los inventarios de vegetación actual realizados. Lógicamente, es necesario tener en cuenta las series previamente definidas para el territorio biogeográfico estudiado, realizando las modificaciones precisas a la luz de la información propia. En este sentido, destaca la obra de carácter general (a escala 1/400.000) de las series de toda España, de Rivas Martinez et al. (1987).

$3^{a}$ - Interpretación de la degradación que cada formación vegetal representa dentro de su serie correspondiente, mediante la información sobre la estructura y biomasa de dichas formaciones vegetales y sobre los requerimientos ecológicos de las especies significativas de las mismas. Se definen las etapas de degradación ${ }^{7}$ a que han dado lugar los distintos mecanismos de degeneración que han modificado la comunidad climácica de cada serie.

$4^{\mathrm{a}}$ - Planteamiento de una hipótesis sobre la distribución territorial de las series previamente definidas, confeccionando el Mapa de Series de Vegetación a partir de la información espacial de los factores que las condicionan. Esta hipótesis se confirma analizando, para cada punto de muestreo, si el inventario florístico existente puede pertenecer a la serie de vegetación adjudicada según la hipótesis de trabajo. Un sencillo contraste estadístico permite confirmar o rechazar la delimitación

\footnotetext{
${ }^{6}$ La serie de vegetación hace referencia al conjunto de comunidades vegetales que pueden encontrarse en un espacio de características ambientales homogéneas, como resultado del proceso de la sucesión; es decir, reúne tanto la formación vegetal más próxima a la hipotética clímax como aquellas correspondientes a las distintas etapas de degradación que la reemplazan.

${ }^{7}$ Dependiendo de los objetivos del trabajo, se puede ser más o menos minucioso en la definición de las etapas de degradación, pero a estas escalas, para las series climácicas, es posible diferenciar bien 7 etapas (1- bosque denso; 2- bosque poco denso; 3- bosque claro o matorral arbustivo con árboles aislados; 4- matorral denso; 5- matorral degradado y repoblaciones con especies no pertenencientes a la serie climácica; 6- pastizal; 7-cultivos). En las series edafófilas, resulta difícil diferenciar más de 2 etapas.
} 
cartográfica realizada, ya sea globalmente o para las distintas unidades de paisaje. La validez de esa delimitación supone, en definitiva, una demostración del papel que los factores determinantes de la presencia de esas Series (climáticos, edáficos...) juegan como factores explicativos del paisaje.

$5^{\text {a }}$ - Planteamiento de diversas hipótesis explicativas de las etapas de degradación actual de la vegetación en relación a la influencia de los factores antrópicos fundamentalmente. Tras definir bien cada una de las variables (en gran parte categóricas) y la forma en que intervienen en cada una de las hipótesis, deben realizarse los contrastes estadísticos adecuados para su confirmación o rechazo. Ello puede hacerse relacionando las etapas de degradación existentes (variable dependiente) con la presencia de los distintos factores explicativos (variables independientes), utilizando para ello la información recogida en los puntos de muestreo.

\section{DIAGNOSTICO SOBRE LA DINAMICA DEL PAISAJE (FASE IV)}

La dinámica del paisaje depende de la conjunción de todos los factores que determinan el fenosistema (geológicos, climáticos y antrópicos), siendo su diagnóstico un objetivo difícil de alcanzar en toda su complejidad. Sin embargo, pensamos que es posible y tiene interés emitir un diagnóstico "aproximativo" sobre la evolución o dinámica de cada una de las unidades de paisaje delimitadas, utilizando la información generada en las etapas anteriores y con un método sencillo:

En primer lugar, se propone determinar la situación de degradación en que se encuentra cada unidad ambiental, en conjunto, respecto al teórico clímax de su correspondiente serie de vegetación. Esto se consigue mediante un análisis de las frecuencias de cada etapa de degradación dentro de cada unidad. De igual manera, puede establecerse la posición de las unidades en otras escalas de degradación ambiental.

A continuación, se aborda el diagnóstico de la dinámica de la vegetación. Entendemos por ésta el sentido de su evolución respecto al teórico clímax de la serie de vegetación a la que corresponda. La dinámica es progresiva cuando, partiendo de cualquier situación de degradación, la evolución de una formación vegetal determinada tiende a aproximarla a la situación climácica. Por el contrario, es regresiva cuando tiende a alejar a dicha formación de la situación climácica, empobreciéndose cualitativa y cuantitativamente. Por último, se habla de "estabilidad" cuando la tendencia es a la permanencia en la situación de degradación actual. En cualquiera de los tres casos, las tendencias que pueden observarse y 
diagnosticarse, en base a lo observado en los últimos años, son siempre transitorias, pues pueden variar en el momento (muchas veces imprevisible) en que lo haga cualquiera de los factores influyentes.

La tendencia evolutiva de la vegetación se evalúa para cada uno de los puntos muestreados siendo fundamental averiguar la etapa existente previamente en cada punto (remontándose hasta un máximo de 3 ó 4 décadas, normalmente), mediante fotografía aérea antigua, testimonios directos, documentación de archivos, fotografías... Es precisa, además, información sobre las variables que, según se haya deducido de los análisis anteriores, intervengan decisivamente en los procesos de degradación del espacio estudiado. Entre estas variables, cabe destacar la existencia de incendios forestales, de plagas en el arbolado, la intensidad de los distintos aprovechamientos del monte (carga ganadera, leña, caza...), el grado de presencia humana, la accesibilidad, etc...

Según los criterios expuestos, y tras haber relacionado las variables citadas, se puede emitir un diagnóstico sobre la dinámica vegetal en cada uno de los puntos del muestro y, en consecuencia, mediante una sencilla tabla de frecuencias, obtener un diagnóstico global para cada una de las unidades de paisaje, lo que sin duda puede tener gran aplicación, especialmente en la gestión de áreas con interés naturalístico.

En tercer lugar, podemos aproximarnos al balance morfogenético que es, en realidad, un balance entre morfogénesis y edafogénesis, pues se trata de diagnosticar cuál de estos procesos predomina, en conjunto, en las distintas unidades de paisaje. Cuando los procesos de meteorización dominan, el balance morfogenético es negativo, siendo la génesis de alteritas y de suelo el proceso fundamental, lo que supone un medio en biostasia. Si son los procesos de ablación y de transporte los que dominan, evacuando las alteritas y suelos desarrollados, la morfogénesis es activa y se habla de un medio en rexistasia. Existen otras situaciones denominadas intergrados (Tricart y Kilian, 1979), en las que el balance puede ser favorable a la edafogénesis o a la morfogénesis, pero muy débilmente; son medios en biostasia relativa o rexistasia relativa.

Los factores que intervienen en este balance y que hay que concretar en variables son los siguientes: Factores activos: clima, acción marina, cubierta vegetal; Factores pasivos: litología, pendiente, dispositivo estructural, grado de fracturación... Estos factores deben concretarse en variables con distintas categorías a las se que aplicará un valor índice que aumente a medida que favorezca más la morfogénesis. Caracterizando las unidades de paisaje según cada variable y adjudicándoles el valor índice correspondiente, se construye una tabla que permite valorar el peso de los distintos factores y emitir un diagnóstico sobre su situación de biostasia o rexistasia. 
La dinámica global para cada una de las unidades de paisaje se establece combinando la situación de degradación actual de la vegetación, el sentido de su evolución respecto a la vegetación climácica y el balance entre morfogénesis y edafogénesis.

\section{BIBLIOGRAFIA}

BARSCH, D. (1963): "Stdiem zum problem der deformation von baumkronen durch wind", Freiburger Geographische Hefte, 1.

BERTRAND, G. (1966): "Pour un étudee géographique de la végétation", Revue Géographique des Pyrénées et du Sud-Ouest, 37, 129-135.

BERTRAND, G. (1966): "Pour un étudee géographique de la végétation", Revue Géographique des Pyrénées et du Sud-Ouest, 37, 129-135.

BERTRAND, G. (1968): "Paysage et Géographie physique globale. Esquisse méthodologique", Revue Géographique des Pyrénées et du Sud-Ouest, 39, 249-271.

BERTRAND, G. (1969): "Ecologie de l'espace géographique". C. R. Soc. Biogéographique, 406, 195-205.

BERTRAND, G. (1972): "Les estructures naturelles de l'espace géographique. L'exemple des Montagnes Cantabriques centrales", Revue Géographique des Pyrénées et du Sud-Ouest, $43,175-206$.

BERTRAND, G. y BERUTCHACHVILI, N. (1978): "Le géosystème ou système territoriel naturel", Revue Géographique des Pyrénées et du Sud-Ouest, 49, 167-180.

BRAUM BLANQUET, J. (1979): Fitosociología, Blume, Madrid.

DENNESS, B. y GRAINGER, P. (1976): "The preparation of slope maps by the movil interval method", Area, 18, 213-219.

EHRART, H. (1956): La genèse des sols en tant que phénomène géologique, Ed. Masson, París.

GONZALEZ BERNALDEZ, F. (1981): Ecología y paisaje, Blume, Madrid.

HAASE, G. (1964): "Landschaftsökologische Detailuntersuchung und naturräumliche Gliederung", Pertermanns geographisch Mitteilungen, 108, 8-30. 
IBARRA, P. (1993): Naturaleza y hombre en el Sur del Campo de Gibraltar: un análisis paisajístico integrado, Ed. Junta de Andalucía, Agencia de Medio ambiente, Sevilla.

ISACHENKO, A. G. (1978): Introducción al estudio de los geosistemas, Novosibirsk, Nauka.

MORISAVA, M.E. (1985): Rivers form and process Geomorphology, Texs 7, Longman, London.

NEEF, E. (1963): Topologische und chorologische Arbeitsweisen in der Landschaftsforschung, Peter. Geogr.

RIVAS MARTINEZ, S. et al. (1987): Memoria del Mapa de Series de vegetación de España, $\mathrm{M}^{\circ}$ de Agricultura, Pesca y Alimentación, Madrid.

SOCHAVA, V.B. (1963): Opriedielieni niekotorykh ponyatii i terminov fizicheskoi gheografii (Definición de algunos conceptos y términos de geografía física), Doklady In-ta gheografii Sibiri i Dal'niego Vostoka.Vyp. 3.

TANSLEY, (1935).: The use and abuse of vegetational concepts and terms, Ecology $16,284-307$.

TRICART, J. y CAILLEUX, A. (1956): "Le problème de la classification des faits géomorphologiques", Annales Géogaph., LXV, 162-186.

TRICART, J. y KILIAN, J. (1979): L'éco-géographie et l'aménagement du milieu naturel, Ed. Maspero, París.

TROLL, C. (1939): "Luftbildplan und ökologische Bodenforschung", Z. Ges. Erdk. Berl., 241-298.

TROLL, C. (1950): "Die geographische Landschaft und ihre Erforschung", Studium Gen., 3, 163181. 